\title{
The Effect of Perceptual-Motor Exercise on Improvement in Executive Functions of Children with Autism Disorder
}

\author{
Hadi Moradi $^{*}$, Ahmadreza Movahedi², Mohadeseh Arabi ${ }^{3}$ \\ ${ }^{1}$ Department of Motor Behavior, Faculty of Sport Sciences, Ferdowsi University of Mashhad, Mashhad, Iran \\ ${ }^{2}$ Department of Motor Behavior, Faculty of Sport Sciences, University of Isfahan, Isfahan, Iran \\ ${ }^{3}$ Faculty of Literature and Humanities, Najafabad Branch, Islamic Azad University, Najafabad, Iran
}

\section{ABSTRACT}

Introduction: Autism Spectrum Disorder is a neurodevelopmental disorder characterized by impairment in social interaction and communication, accompanied by stereotyped and repetitive behavior. The purpose of the present study was to investigate the effect of a perceptual-motor exercise period on executive functions (neurocognitive functions of attention) of children with autism disorder in the age group of 7-12 years. Materials and Methods: The method of conducting the present study was semi-experimental. Among children with autism disorder referring to autistic children's care centers in Isfahan, 30 patients were selected using screening tests and after measuring neuro-cognitive functions of attention by neuropsychological Conner's questionnaire as a pretest, randomly divided into two experimental $(n=15)$ and control $(n=15)$ groups. In the experimental group, perceptualmotor exercises were performed for 12 weeks and 3 sessions weekly. Finally, a post-test was performed for all of the subjects. Results: The results have shown that perceptual-motor exercise in the experimental group was an effective measure to improve the neurocognitive functions of the attention of children with autism disorder. Besides, neuro-cognitive functions of attention were significantly better in the experimental group compared to the control group. Conclusion: Perceptual-motor exercises with music may provide appropriate intervention for improving the neurocognitive functions of the attention of children with autism disorder.

\section{Key words:}

1. Autism Spectrum Disorder

2. Exercise

3. Attention

*Corresponding Author: Hadi Moradi

E-mail: hadi.moradi@stu.um.ac.ir 
تأثير تمرينات ادراكى -حركتى بر بهبود كاركردهاى اجرايى كودكان مبتلا به اختلال اوتيسم

\author{
هادى مرادى"'، احمدرضا موحدى'، محدثه عربى" \\ 'كروه رفتار حركتى، دانشكده علوم ورزشى، دانشكاه فردوسى مشهد، مشهد، ايران

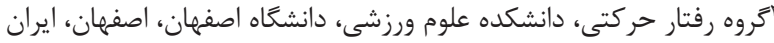 \\ "دانشكده ادبيات و علوم انسانى، واحد نجف آباد، دانشكاه آزاد اسلامى، نجف آباد، ايران
}

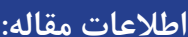

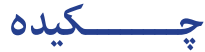

مقدمه: اختلال طيف اوتيسم يك اختلال عصبى است كه به وسيلة اختلال در تعاملات اجتماعى و

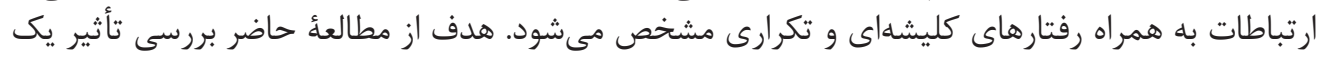

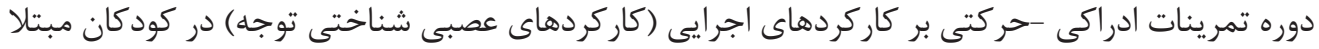

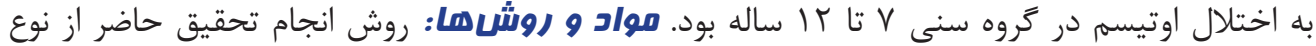

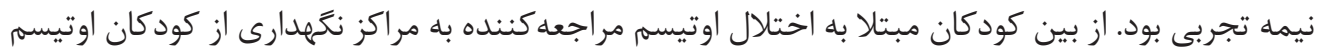

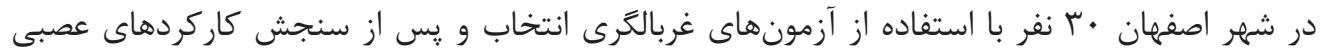

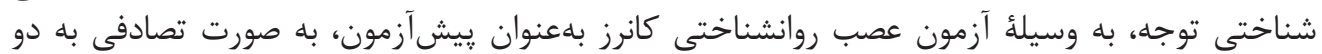

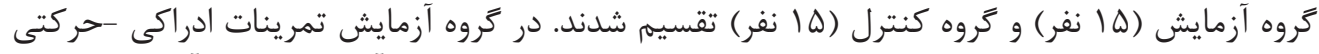

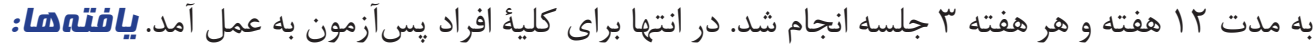

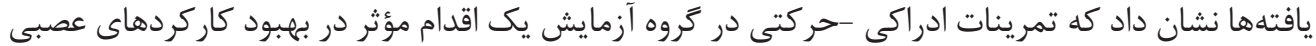

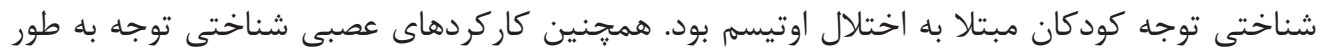

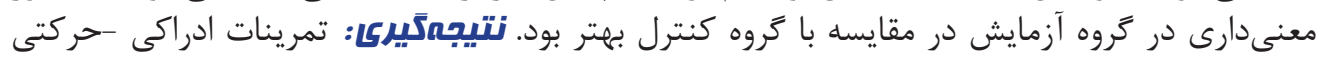

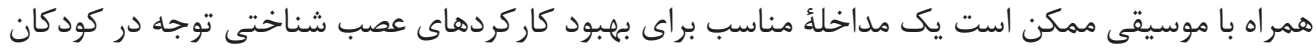

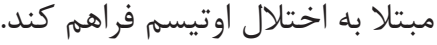

كليد وازهها:

ا.

r. r.

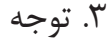

" نويسنده مسئول: هادى مرادى

آدرس الكترونيكى: hadi.moradi@stu.um.ac.ir 


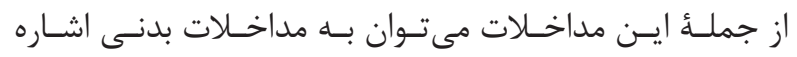

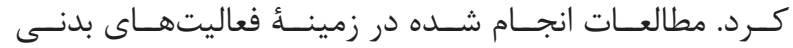

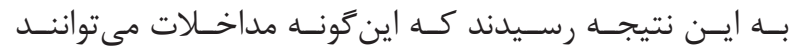

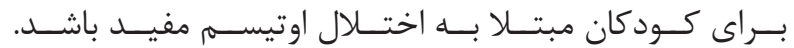

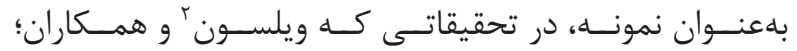

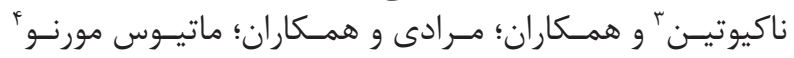

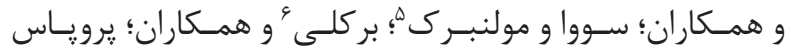

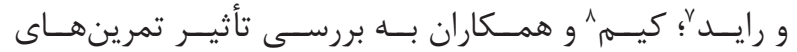

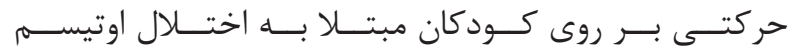

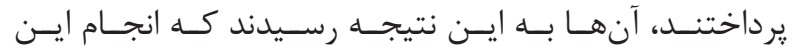

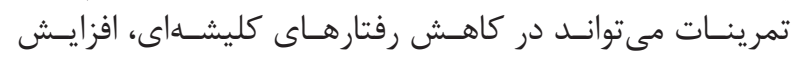

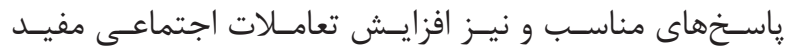

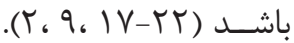

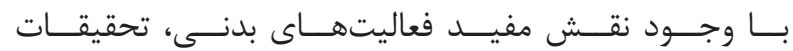

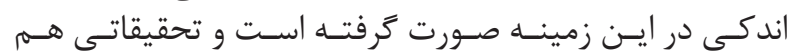

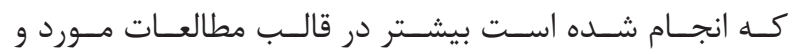

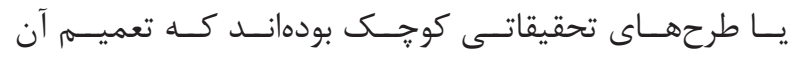

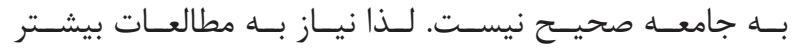

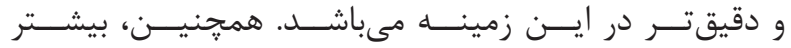

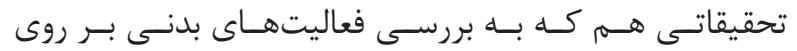

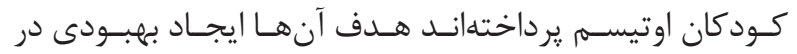

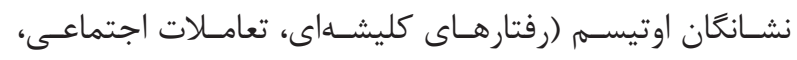

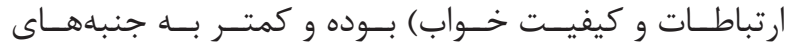

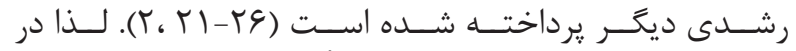

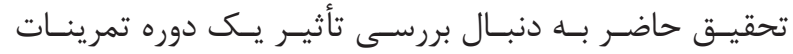

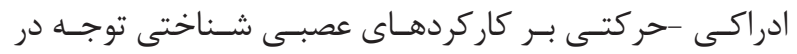

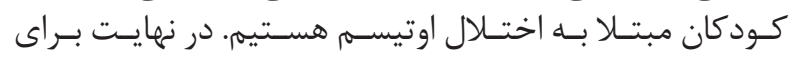

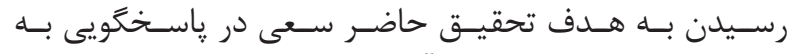

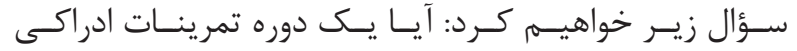

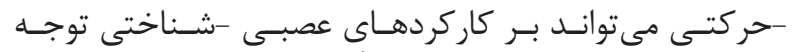

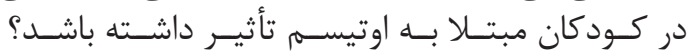

\section{مواد و روشها - - ماد}

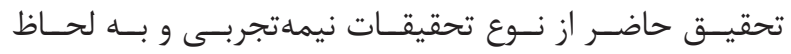

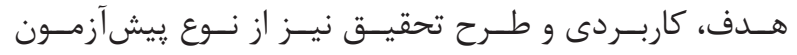

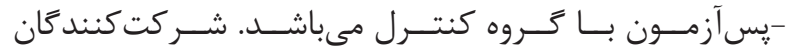

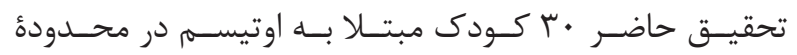

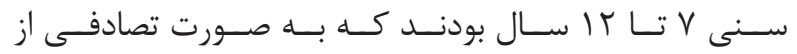

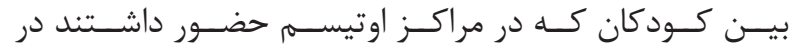

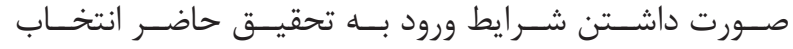

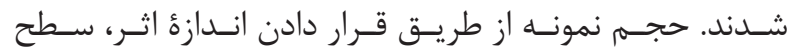

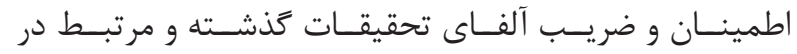

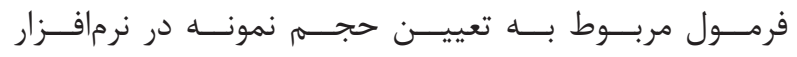

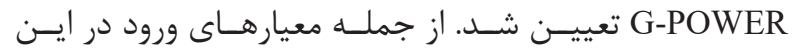

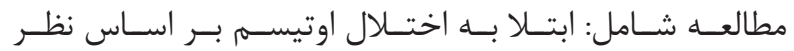

${ }^{1}$ Green

${ }^{2}$ Wilson

${ }^{3}$ Nakutin

${ }^{4}$ Mateos-Moreno
مقدمه

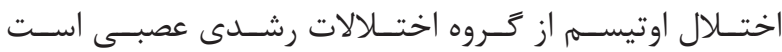

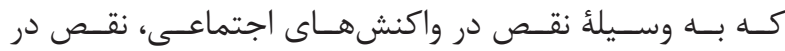

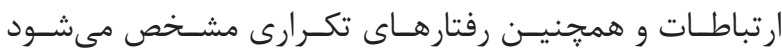

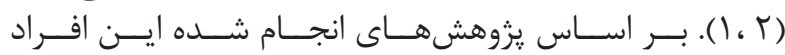

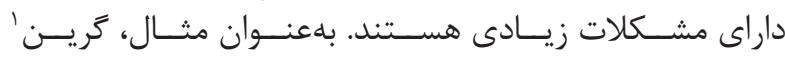

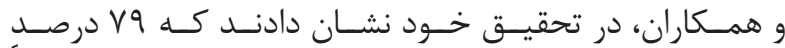

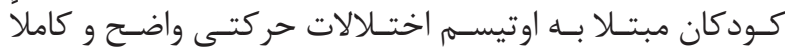

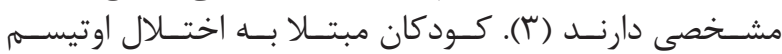

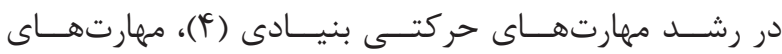

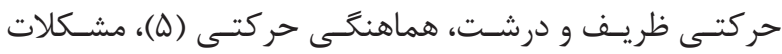

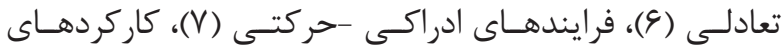

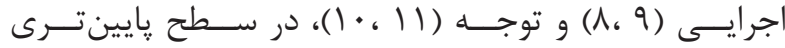

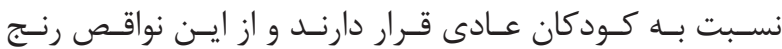
مى برنــد.

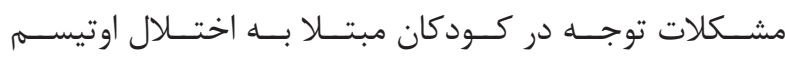

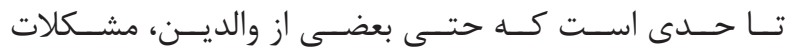

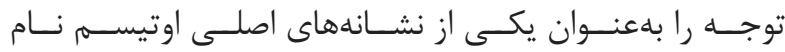

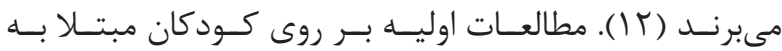

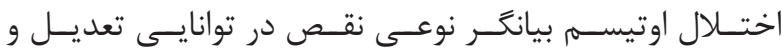

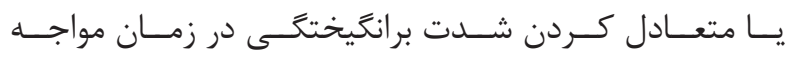

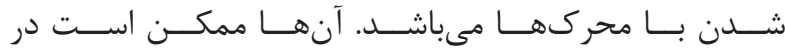

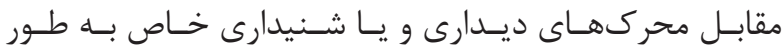

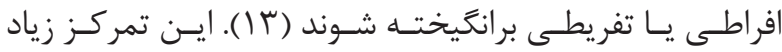

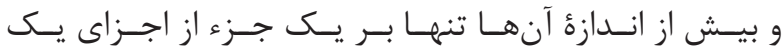

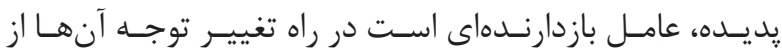

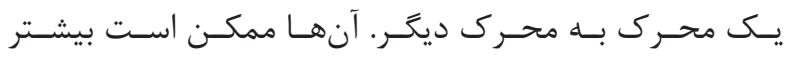

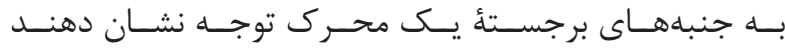

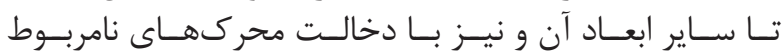

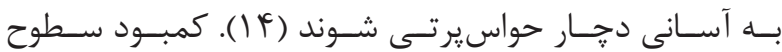

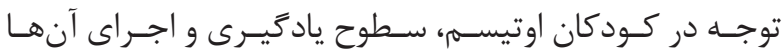

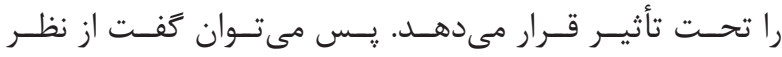

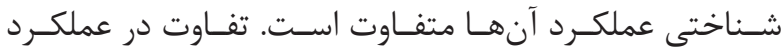

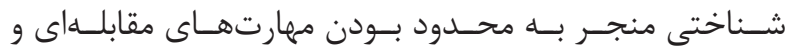

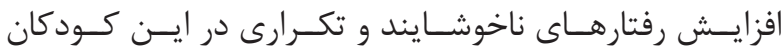

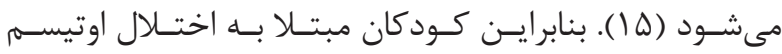

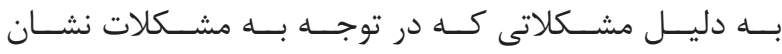

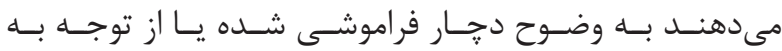

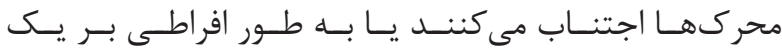

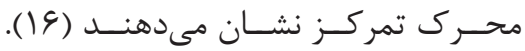

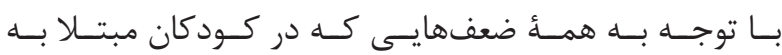

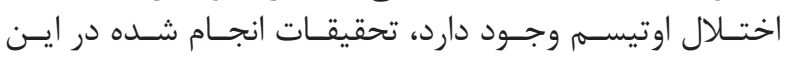

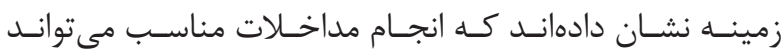

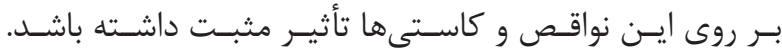

${ }^{5}$ Sowa and Meulenbroek

${ }^{6}$ Berkeley

${ }^{7}$ Prupas and Reid

${ }^{8}$ Kern 


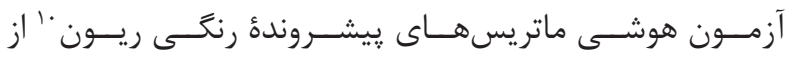

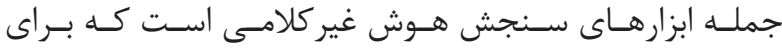

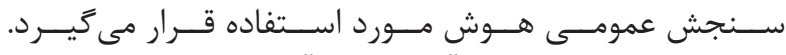

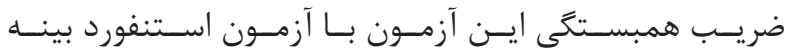

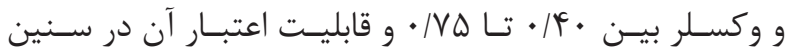

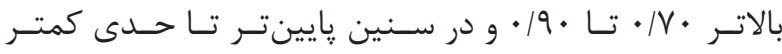

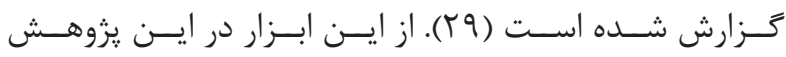

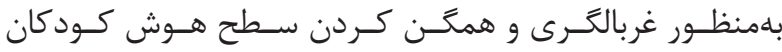

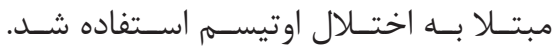

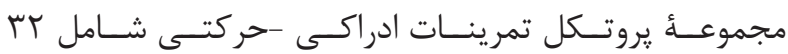

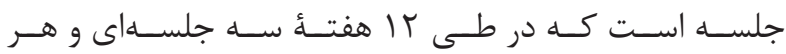

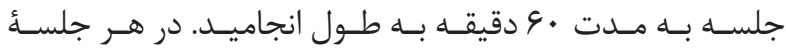

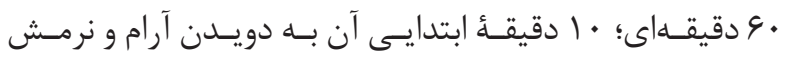

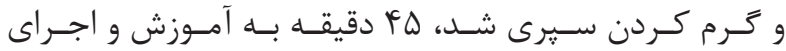

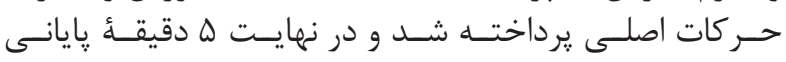

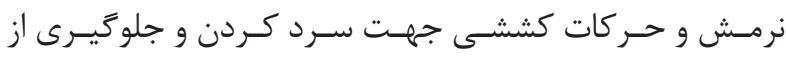

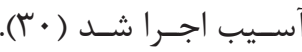

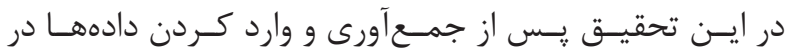

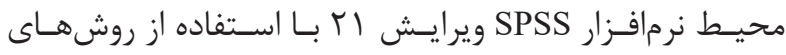

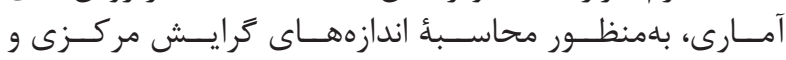

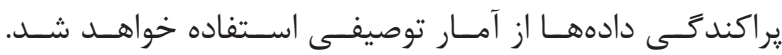

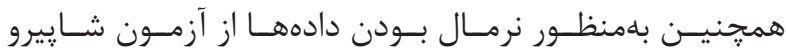

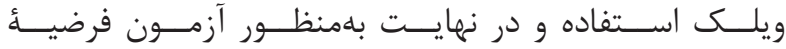

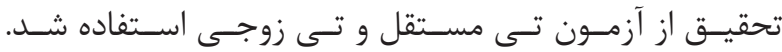

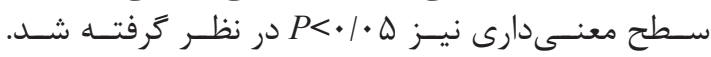

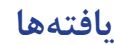

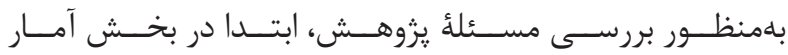

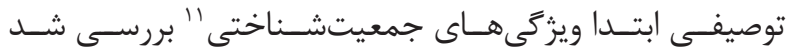

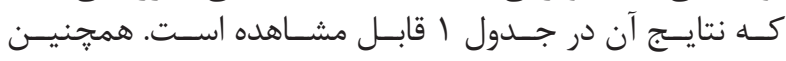

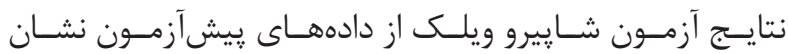

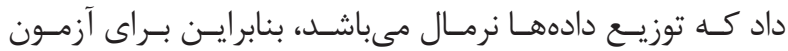

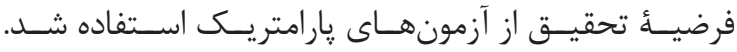

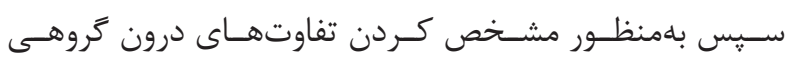

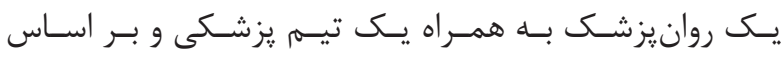

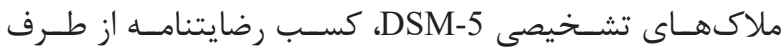

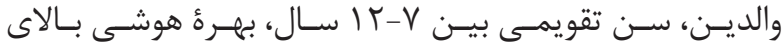

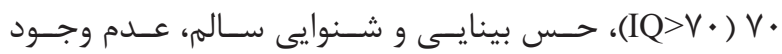

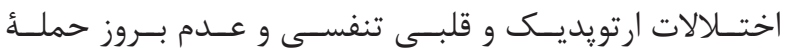

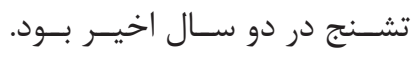

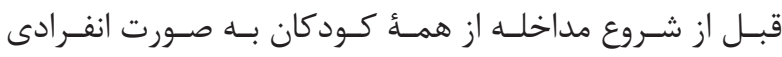

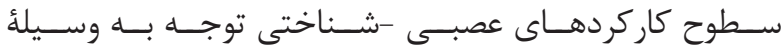

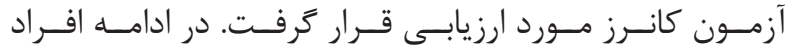

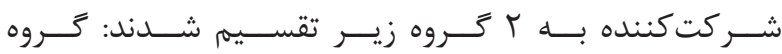

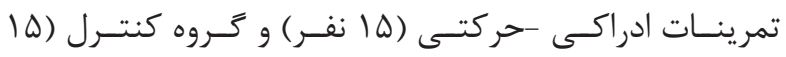

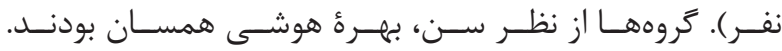

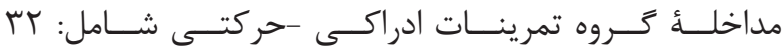

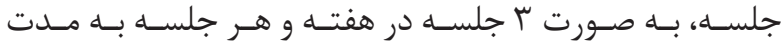

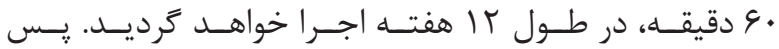

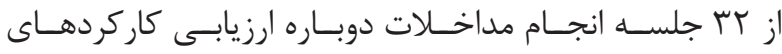

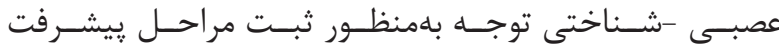

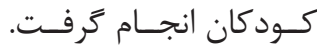

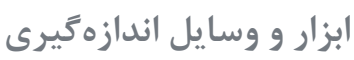
آزمون كانرز

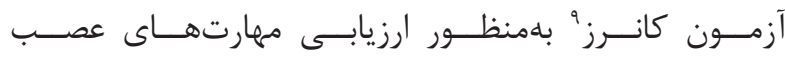

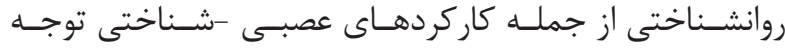

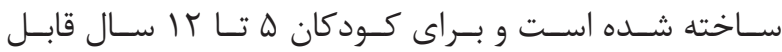

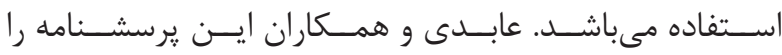

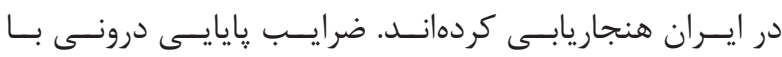

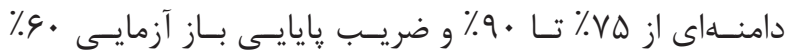

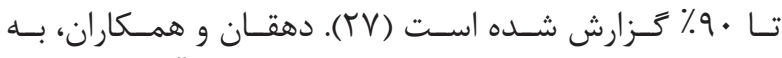

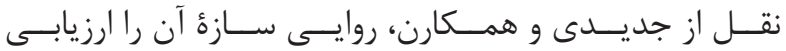

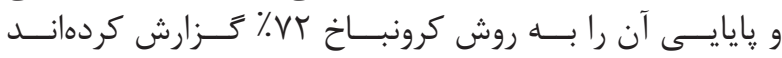

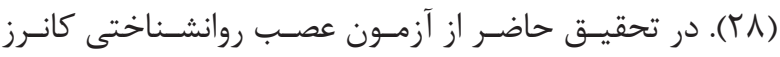

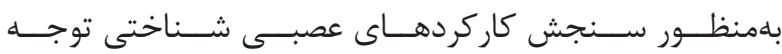

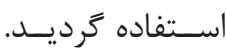

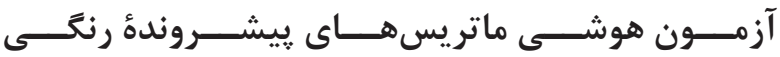

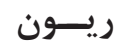

جدول ا- بِيشآزمون و پِسآزمون متغيرهاى تحقيق و مشخصات شركت كندكان در كروههاى كنترل و كَروه آزمايش.

\begin{tabular}{|c|c|c|c|}
\hline 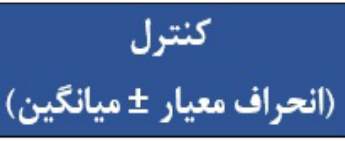 & (انحراف معيار \ ميانكين) & \multicolumn{2}{|r|}{ كروه } \\
\hline ل الفر & ل الفر & \multicolumn{2}{|r|}{ تعداد } \\
\hline $9 / r r \pm 1 / r r$ & $\Lambda / q r \pm 1 / \Delta r$ & \multicolumn{2}{|c|}{ سن (سال) } \\
\hline$q \Delta / \Delta r \pm V / q F$ & $q r / 9 V \pm q / r r$ & \multicolumn{2}{|c|}{ IQ } \\
\hline $\mid F / r r \pm r / r \Delta$ & $1 Q / \Lambda V \pm r / V V$ & بيش آزمون & كاركردهاى عصبى \\
\hline $\mid r / f \cdot \pm r / r q$ & $1 . / 9 r \pm T / r \mid$ & يس آزمون & -شناختى توجه \\
\hline
\end{tabular}

${ }^{9}$ Canners

${ }^{10}$ Test de matrizes progressivas de raven

${ }^{11}$ Demographic 


$$
\text { س ارائــهـ شـده اسـتـ. }
$$

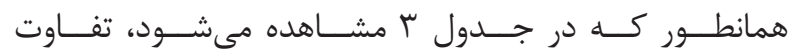

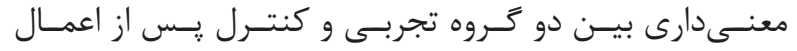

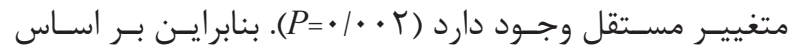

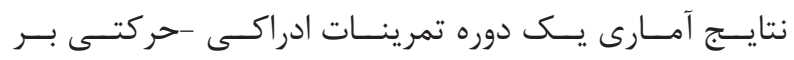

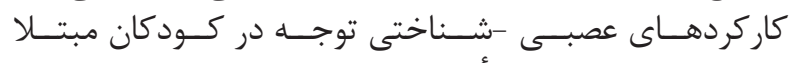

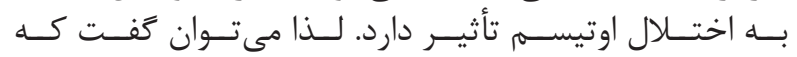

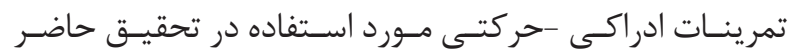

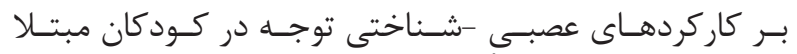

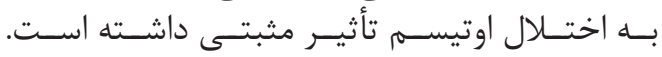

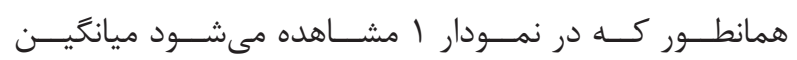

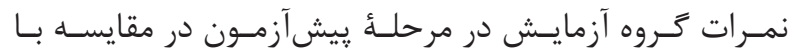

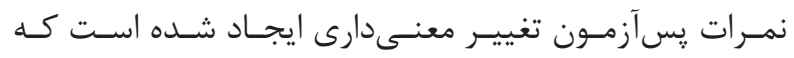

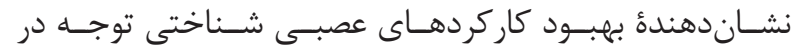

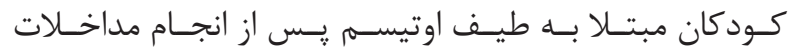

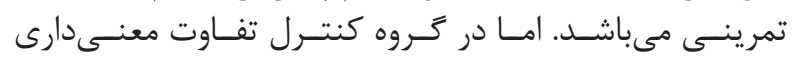
مشـاهده نشـده اسـت (نمــودار (1).

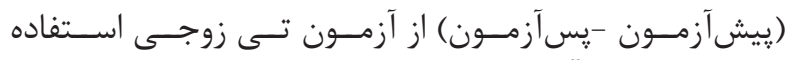

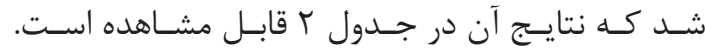

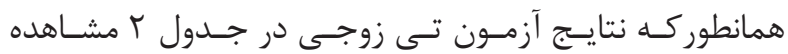

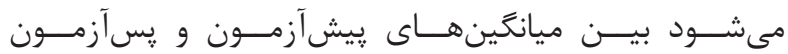

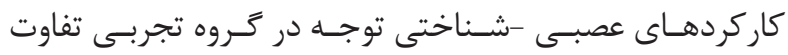

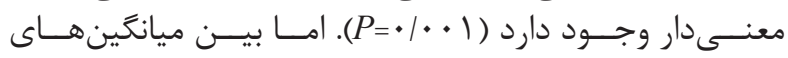

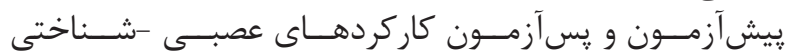

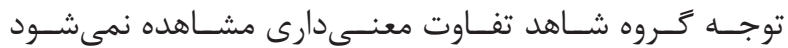
. $\left(P=\cdot \mid \wedge \boldsymbol{\Upsilon}^{\mathrm{N}}\right)$

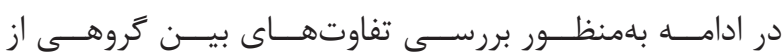

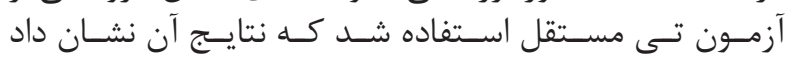

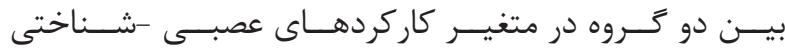

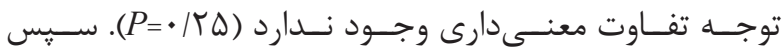

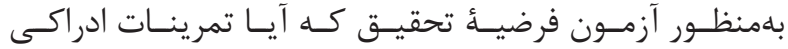

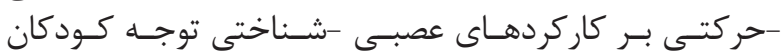

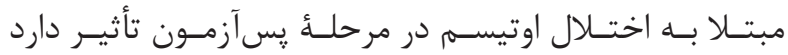

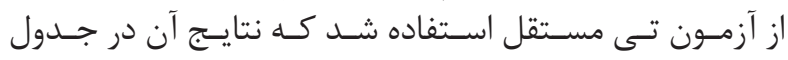

جدول r- نتايج آزمون t زوجى مربوط به كاركردهاى عصبى -شناختى توجه در كروههاى كنترل و تجربى (معنى دارى **).

\begin{tabular}{|c|c|c|c|c|}
\hline سطح معنى دارى & مقدار t & نمرات كاركردهاى عصبى -شناختى توجه & مرحله & كروه - مره \\
\hline \multirow{2}{*}{$\cdot \mid \Lambda \mu$} & \multirow{2}{*}{$\cdot|r| 1$} & $\mid f / r r \pm r / r \Delta$ & ي بيش آزمون & \multirow{2}{*}{ 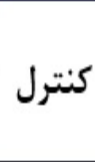 } \\
\hline & & $\mid F / F+ \pm r / r q$ & 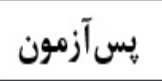 & \\
\hline \multirow{2}{*}{$\cdot 1 \cdot .1^{*}$} & \multirow{2}{*}{$4 / T F$} & $\mid Q / \Lambda V \pm Y / V V$ & بيش آزمون & \multirow{2}{*}{ تجربى } \\
\hline & & $1 \cdot / 9 r \pm r / r \mid$ & يسآزمون & \\
\hline
\end{tabular}

جدول ب- نتايج آزمون t مستقل مربوط به كاركردهاى عصبى -شناختى توجه در كروههاى كنترل و تجربى (معنى دارى *).

\begin{tabular}{|c|c|c|c|c|}
\hline سطح معنىدارى & درجه آزادى & مقدار t & مرحله & متغير وابسته \\
\hline$\cdot / r \Delta$ & rᄉ & $1 / 1 \mathrm{~V}$ & پيش آزمون & \multirow{2}{*}{ كاركردهاى عصبى -شناختى } \\
\hline $.1 \cdot r *$ & $r \Lambda$ & r/T & يسآزمون & \\
\hline
\end{tabular}

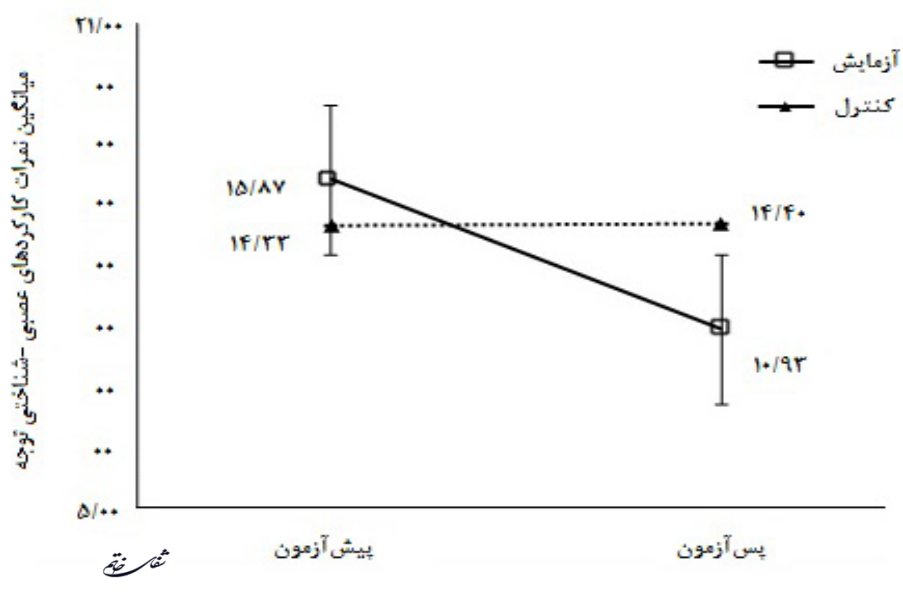




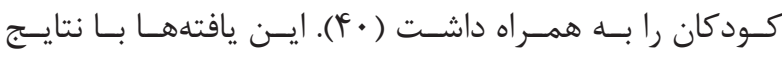

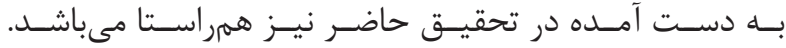

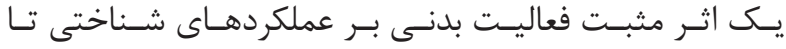

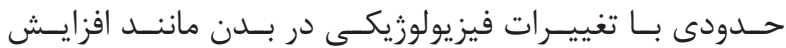

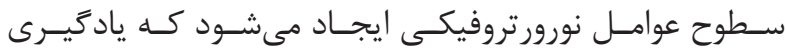

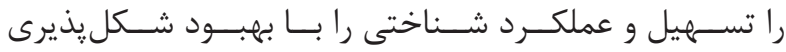

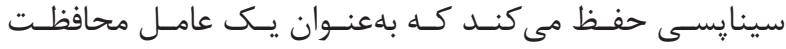

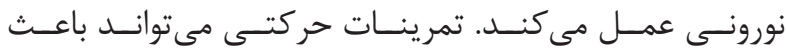

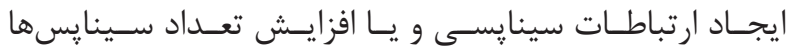

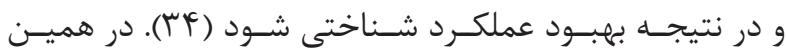

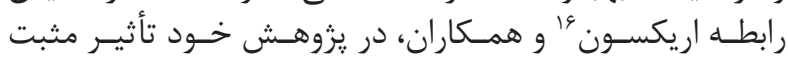

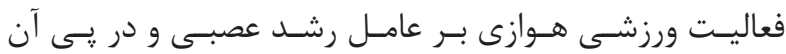

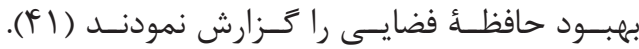

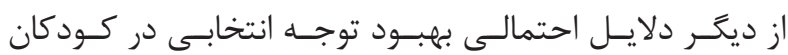

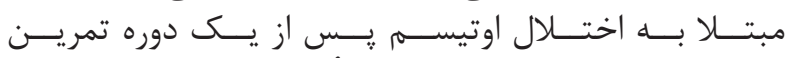

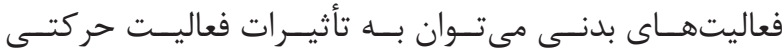

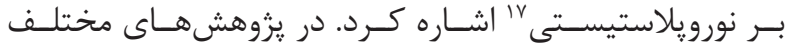

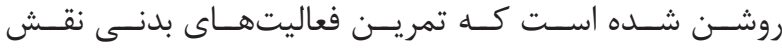

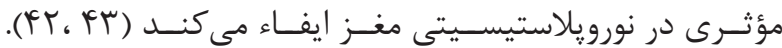

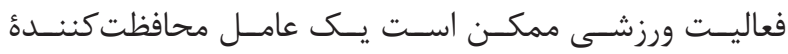

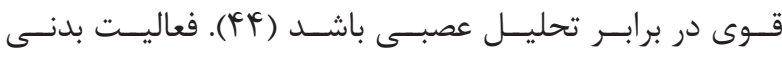

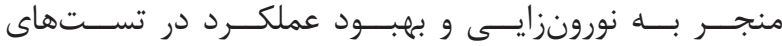

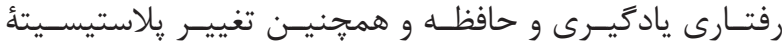

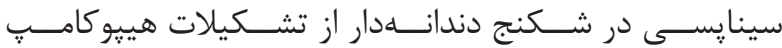

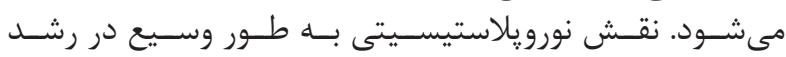

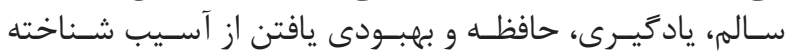

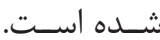

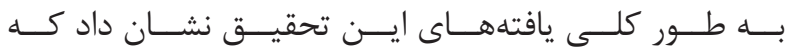

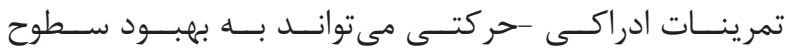

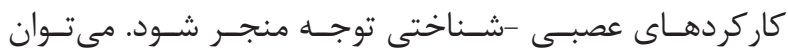

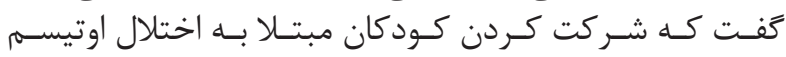

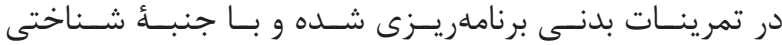

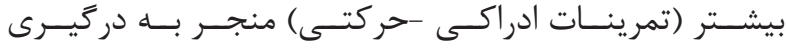

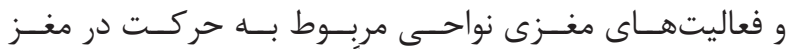

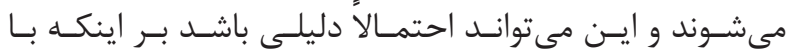

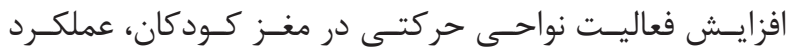

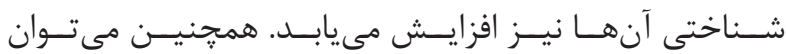

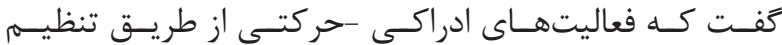

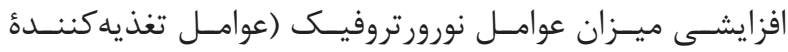

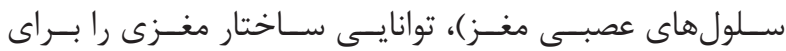

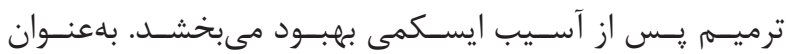

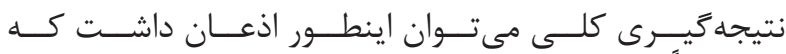

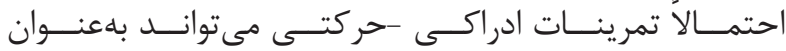

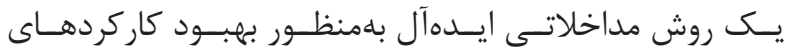

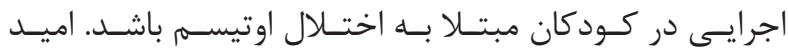

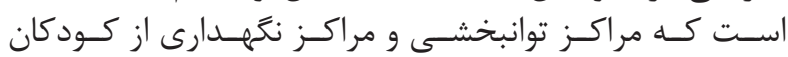

${ }^{12}$ Pan

${ }^{13}$ Bremer

${ }^{14}$ Hilton
بحث و نتيجه كيرى

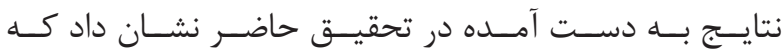

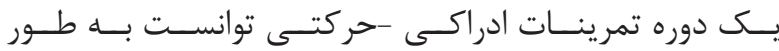

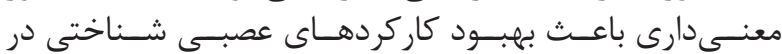

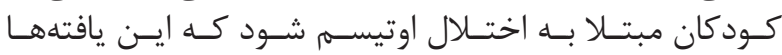

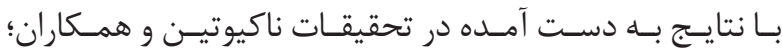

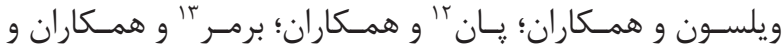

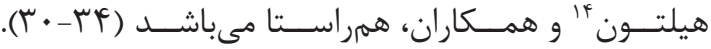

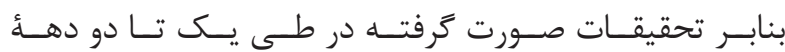

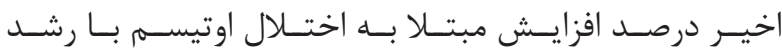

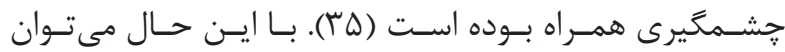

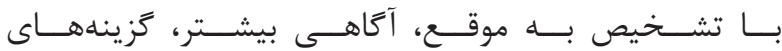

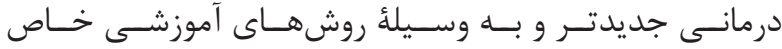

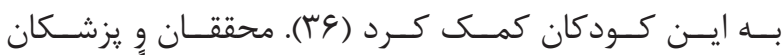

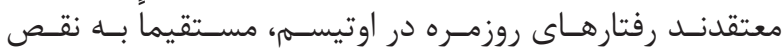

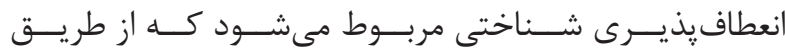

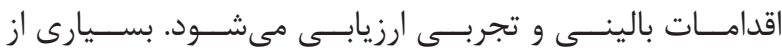

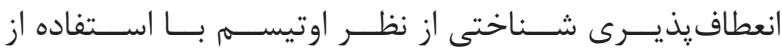

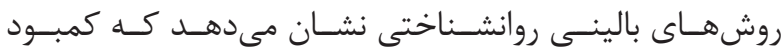

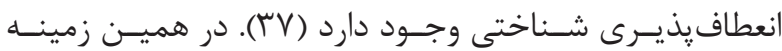

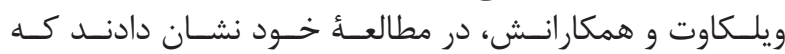

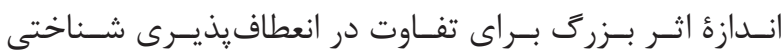

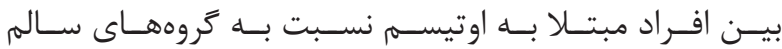

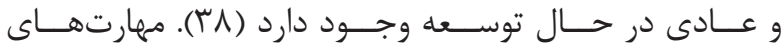

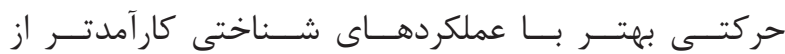

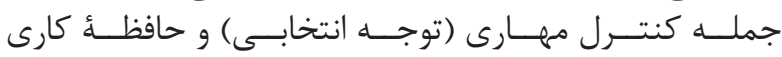

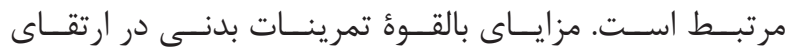

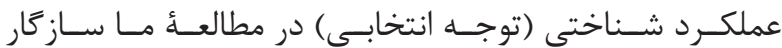

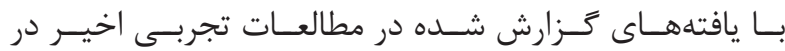

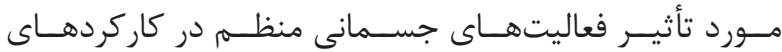

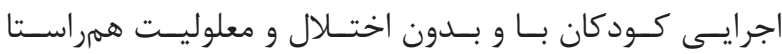

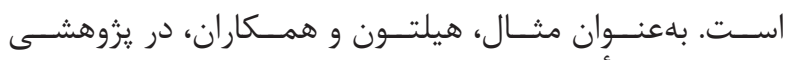

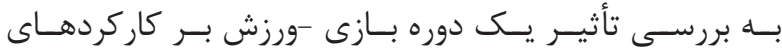

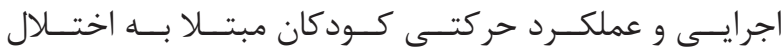

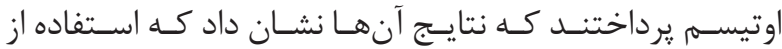

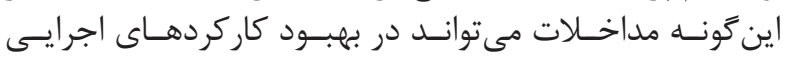

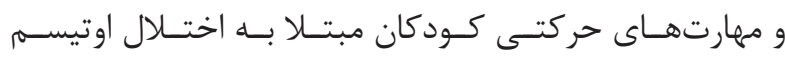

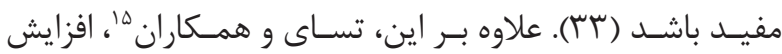

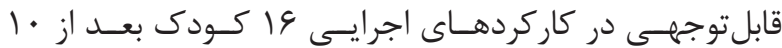

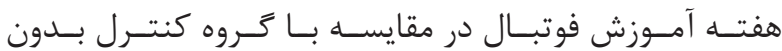

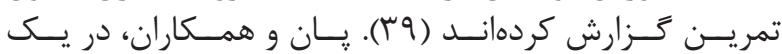

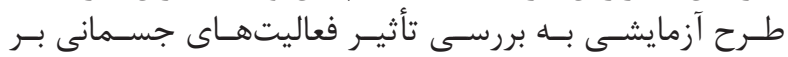

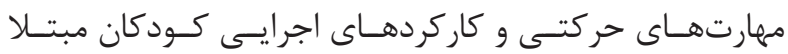

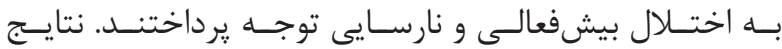

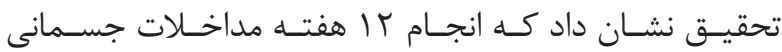

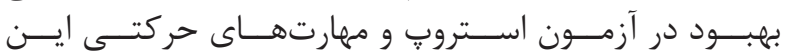

${ }^{15}$ Tsai

${ }^{16}$ Erickson

${ }^{17}$ Neuroplasticity 


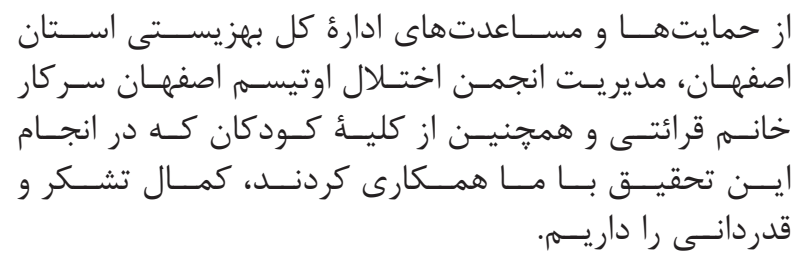

1. Association AP. Diagnostic and statistical manual of mental disorders (DSM-5®): American Psychiatric Pub. 2013.

2. Moradi H, Sohrabi M, Taheri H, Khodashenas E, Movahedi A. Comparison of the effects of perceptual-motor exercises, vitamin D supplementation and the combination of these interventions on decreasing stereotypical behavior in children with autism disorder. International Journal of Developmental Disabilities. 2018; 1-10.

3. Green D, Charman T, Pickles A, Chandler S, Loucas $\mathrm{T}$, Simonoff E, et al. Impairment in movement skills of children with autistic spectrum disorders. Developmental Medicine \& Child Neurology. 2009; 51(4): 311-6.

4. Morris SL, Foster CJ, Parsons R, Falkmer M, Falkmer T, Rosalie SM. Differences in the use of vision and proprioception for postural control in autism spectrum disorder. Neuroscience. 2015; 307: 273-80.

5. Fournier KA, Hass CJ, Naik SK, Lodha N, Cauraugh $\mathrm{JH}$. Motor coordination in autism spectrum disorders: a synthesis and meta-analysis. Journal of Autism and Developmental Disorders. 2010; 40(10): 1227-40.

6. Liu T, Breslin CM. Fine and gross motor performance of the MABC-2 by children with autism spectrum disorder and typically developing children. Research in Autism Spectrum Disorders. 2013; 7(10): 1244-9.

7. Jepsen RH, VonThaden K. The effect of cognitive education on the performance of students with neurological developmental disabilities. NeuroRehabilitation. 2002; 17(3): 201-9.

8. Chan AS, Cheung M-c, Han YM, Sze SL, Leung WW, Man HS, et al. Executive function deficits and neural discordance in children with autism spectrum disorders. Clinical Neurophysiology. 2009; 120(6): 1107-15.

9. Nakutin SN, Gutierrez G, Campbell J. Effect of physical activity on academic engagement and executive functioning in children with ASD. School Psychology Review. 2019; 48(2): 177-84.

10. Afshari J. The effect of perceptual-motor training on

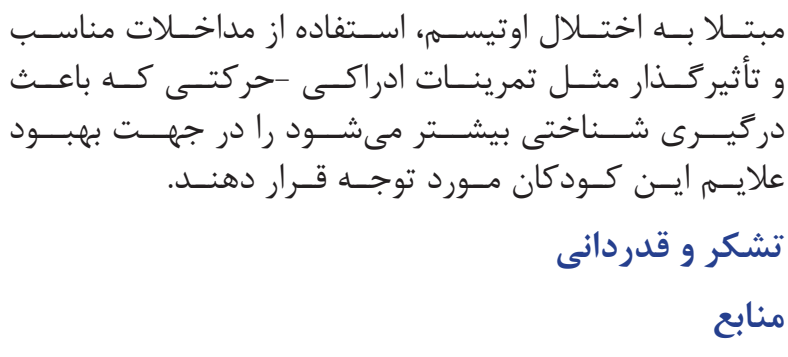

attention in the children with autism spectrum disorders. Research in Autism Spectrum Disorders. 2012; 6(4): 1331-6.

11. Tse CYA, Lee HP, Chan KSK, Edgar BV, WilkinsonSmith A, Lai WHE. Examining the impact of physical activity on sleep quality and executive functions in children with autism spectrum disorder: A randomized controlled trial. Autism. 2019.

12. Ornitz EM. Autism: A disorder of directed attention. Brain Dysfunction. 1988.

13. Lovaas OI, Koegel RL, Schreibman L. Stimulus overselectivity in autism: A review of research. Psychological Bulletin. 1979; 86(6): 1236-54.

14. Lovaas OI, Litrownik A, Mann R. Response latencies to auditory stimuli in autistic children engaged in self-stimulatory behavior. Behaviour Research and Therapy. 1971; 9(1): 39-49.

15. Murray-Slutsky C, Paris BA. Exploring the spectrum of autism and pervasive developmental disorders. Intervention Strategies: Communication Skill Builders. 2000 .

16. Rafei T. Autism assessment and treatment. Tehran: Danjeh Publication. 2016

17. Berkeley SL, Zittel LL, Pitney LV, Nichols SE. Locomotor and object control skills of children diagnosed with autism. Adapted Physical Activity Quarterly. 2001; 18(4): 405-16.

18. Kern L, Vorndran CM, Hilt A, Ringdahl JE, Adelman BE, Dunlap G. Choice as an intervention to improve behavior: A review of the literature. Journal of Behavioral Education. 1998; 8(2): 151-69.

19. Prupas A, Reid G. Effects of exercise frequency on stereotypic behaviors of children with developmental disabilities. Education and Training in Mental Retardation and Developmental Disabilities. 2001; 36(2): 196-206.

20. Sowa M, Meulenbroek R. Effects of physical exercise on Autism Spectrum Disorders: A meta-analysis. Research in Autism Spectrum Disorders. 2012; 6(1): 46-57. 


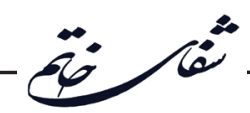

21. Mateos-Moreno D, Atencia-Doña L. Effect of a combined dance/movement and music therapy on young adults diagnosed with severe autism. The Arts in Psychotherapy. 2013; 40(5): 465-72.

22. Wilson KE. The Effect of Swimming Exercise on Amount and Quality of Sleep for Children with Autism Spectrum Disorder. The University of Akron. 2019.

23. Bahrami F, Movahedi A, Marandi SM, Abedi A. Kata techniques training consistently decreases stereotypy in children with autism spectrum disorder. Res Dev Disabil. 2012; 33(4): 1183-93.

24. Fong CE, Jelas ZM. Music education for children with autism in Malaysia. Procedia Soc Behav Sci. 2010; 9: $70-5$.

25. Movahedi A, Bahrami F, Marandi SM, Abedi A. Improvement in social dysfunction of children with autism spectrum disorder following long term Kata techniques training. Research in Autism Spectrum Disorders. 2013; 7(9): 1054-61.

26. Shi Z-M, Lin G-H, Xie Q. Effects of music therapy on mood, language, behavior, and social skills in children with autism: a meta-analysis. Chinese Nursing Research. 2016; 3(3): 137-41.

27. Crews M, Lally J, Gardner-Sood P, Howes O, Bonaccorso S, Smith S, et al. Vitamin D deficiency in first episode psychosis: a case-control study. Schizophrenia Research. 2013; 150(2): 533-7.

28. Murri MB, Respino M, Masotti M, Innamorati M, Mondelli V, Pariante C, et al. Vitamin D and psychosis: mini meta-analysis. Schizophrenia Research. 2013; 150(1): 235-9.

29. Sharifi H. Theory and application of intelligence and personality tests. Tehran: Sokhan Publications. 1997.

30. Moradi H, Sohrabi M, Taheri H, Khodashenas E, Movahedi A. The effects of different combinations of perceptual-motor exercises, music, and vitamin D supplementation on the nerve growth factor in children with high-functioning autism. Complement Ther Clin Pract. 2018; 31: 139-45.

31. Pan C-Y, Chu CH, Tsai CL, Sung MC, Huang CY, Ma WY. The impacts of physical activity intervention on physical and cognitive outcomes in children with autism spectrum disorder. Autism. 2017; 21(2): 190-202.

32. Bremer E, Crozier M, Lloyd M. A systematic review of the behavioural outcomes following exercise interventions for children and youth with autism spectrum disorder. Autism. 2016; 20(8): 899-915.

33. Hilton CL, Cumpata K, Klohr C, Gaetke S, Artner A, Johnson $\mathrm{H}$, et al. Effects of exergaming on executive function and motor skills in children with autism spectrum disorder: a pilot study. Am J Occup Ther. 2014; 68(1): 57-65.

34. Haapala EA. Cardiorespiratory fitness and motor skills in relation to cognition and academic performance in children-a review. Journal of Human Kinetics. 2013; 36(1): 55-68.

35. Cannell JJ. Autism, will vitamin D treat core symptoms? Medical Hypotheses. 2013; 81(2): 195-8.

36. Cannell JJ, Grant WB. What is the role of vitamin D in autism? Dermato-Endocrinology. 2013; 5(1): 199204.

37. Geurts HM, Corbett B, Solomon M. The paradox of cognitive flexibility in autism. Trends in Cognitive Sciences. 2009; 13(2): 74-82.

38. Willcutt EG, Sonuga-Barke EJ, Nigg JT, Sergeant JA. Recent developments in neuropsychological models of childhood psychiatric disorders. Karger Publishers. 2008; P. 195-226.

39. Tsai CL, Wang CH, Tseng YT. Effects of exercise intervention on event-related potential and task performance indices of attention networks in children with developmental coordination disorder. Brain and Cognition. 2012; 79(1): 12-22.

40. Pan CY, Tsai CL, Chu CH, Sung MC, Huang CY, Ma WY. Effects of physical exercise intervention on motor skills and executive functions in children with ADHD: a pilot study. J Atten Disord. 2019; 23(4): 384-97.

41. Erickson KI, Voss MW, Prakash RS, Basak C, Szabo A, Chaddock L, et al. Exercise training increases size of hippocampus and improves memory. Proc Natl Acad Sci U S A. 2011; 108(7): 3017-22.

42. Gomez-Pinilla F. The combined effects of exercise and foods in preventing neurological and cognitive disorders. Preventive Medicine. 2011; 52: S75-S80.

43. Pourtois G, Rauss KS, Vuilleumier P, Schwartz S. Effects of perceptual learning on primary visual cortex activity in humans. Vision Research. 2008; 48(1): 55-62.

44. Griffin ÉW, Mullally S, Foley C, Warmington SA, O’Mara SM, Kelly ÁM. Aerobic exercise improves hippocampal function and increases BDNF in the serum of young adult males. Physiol Behav. 2011; 104(5): 934-41. 